\title{
Alterações hemodinâmicas e intracranianas em cães com hemorragia aguda, anestesiados com isofluorano
}

[Hemodynamic and intracranial alterations in dogs with acute hemorrhage anesthetized with isoflurane]

\author{
C.T. Nishimori ${ }^{1}$, D.P. Paula ${ }^{1}$, P.C. Moraes ${ }^{1}$, E.D.V. Conceição ${ }^{1}$, R. Carareto ${ }^{1}$, \\ N. Nunes ${ }^{2 *}$, P.M.C. Freitas ${ }^{1}$ \\ ${ }^{1}$ Aluna de pós-graduação - FCAV-UNESP \\ ${ }^{2}$ Faculdade de Ciências Agrárias e Veterinárias - UNESP \\ Via de Acesso Prof. Paulo Donato Castellane s/n \\ 14884-900 - Jaboticabal, SP
}

\begin{abstract}
RESUMO
Estudaram-se possíveis alterações hemodinâmicas e intracranianas em cães submetidos à hemorragia aguda e anestesiados pelo isofluorano. Verificou-se também a influência do anestésico no mecanismo de auto-regulação cerebral. Utilizaram-se 20 cães adultos que foram induzidos à anestesia geral com isofluorano por máscara naso-oral a 3,5V\% (volume \%). Após a intubação orotraqueal, reajustou-se o vaporizador para 2,1V\%. Induziuse a hipovolemia retirando-se volume total de $35 \mathrm{ml} / \mathrm{kg}$ de sangue. Avaliaram-se pressão intracraniana (PIC), temperaturas intracraniana (TIC) e corpórea (T), pressão de perfusão cerebral (PPC), pressões arteriais sistólica (PAS), diastólica (PAD) e média (PAM), freqüências cardíaca (FC) e respiratória (FR), índices cardíaco (IC) e sistólico (IS), pressão venosa central (PVC), pressão da artéria pulmonar (PAP), concentração de dióxido de carbono ao final da expiração $\left(\mathrm{ETCO}_{2}\right)$ e saturação de oxihemoglobina $\left(\mathrm{SpO}_{2}\right)$. Imediatamente após a hipovolemia, houve redução significativa da PIC, PPC, PAS, PAD, PAM, IC, IS e PAP. Após 10 minutos, houve aumento gradativo das médias até o final do período experimental. Concluiu-se que a hemorragia aguda promoveu redução das variáveis hemodinâmicas, sendo possível verificar a ativação de mecanismos compensatórios. Além disso, houve redução da perfusão sangüínea e ativação do mecanismo de auto-regulação cerebral, conseqüentes à hipovolemia associada à anestesia com isofluorano.
\end{abstract}

Palavras-chave: cão, hipovolemia, isofluorano, auto-regulação cerebral, hemodinâmica

\begin{abstract}
Intracranial and hemodynamic alterations in 20 adult dogs anesthetized with isoflurane and submitted to acute hemorrhage were studied. Anesthetic influence on cerebral auto-regulation mechanism was also observed. General anesthesia was induced with isoflurane at 3.5V\% (volume \%). Thereafter, orotracheal intubation was performed and vaporizer was calibrated to $2.1 \mathrm{~V} \%$. To induce hypovolemia, a total amount of $35 \mathrm{ml} / \mathrm{kg}^{-1}$ of blood was taken from each dog. Intracranial pressure (ICP); intracranial (ICT) and body temperatures (BT); cerebral perfusion pressure (CPP); systolic (SAP), diastolic (DAP) and mean arterial pressures (MAP); heart $(H R)$ and respiratory rates (RR); cardiac (CI) and stroke indexes (SI); central venous pressure (CVP); pulmonary arterial pressure (PAP); end tidal dioxide carbon $\left(E T C \mathrm{O}_{2}\right)$; and oxyhemoglobin saturation $\left(\mathrm{SpO}_{2}\right)$ were evaluated. Immediately after hypovolemia, there was significative reduction of ICP, CPP, SAP, DAP, MAP, CI, SI, and PAP. Ten minutes after, the values increased gradually until the end of the experimental period. Indeed, acute hemorrhage caused reduction of hemodynamic variables and activation of the compensatory mechanisms. Cerebral blood perfusion was reduced and cerebral auto-regulation mechanism was activated due to hypovolemia associated to isoflurane anesthesia.
\end{abstract}

Keywords: dog, hypovolemia, isoflurane, cerebral auto-regulation, hemodynamic

Recebido em 27 de setembro de 2005

Aceito em 25 de setembro de 2006

*Autor para correspondência (corresponding author)

E-mail: newton@fcav.unesp.br 


\section{INTRODUÇÃO}

A hipovolemia é uma manifestação clínica freqüente na rotina veterinária, principalmente como conseqüência de traumas, sendo um dos sintomas primários dentre as várias manifestações da síndrome do choque.

A síndrome do choque é definida como uma anormalidade do sistema circulatório que resulta em insuficiência da perfusão capilar, prejudicando a oxigenação tecidual. Decorrentes desta hipoperfusão sobrevêm várias alterações funcionais que se somam e, quando não corrigidas, conduzem à irreversibilidade da síndrome (Raiser, 1995). Assim, o conhecimento das alterações fisiológicas que ocorrem durante o choque hemorrágico é fundamental para julgar a gravidade do choque e para a escolha do tratamento adequado, a fim de melhorar o prognóstico do paciente (Meletti e Módolo, 2003).

Segundo Clarck (1992), em situações de hemorragia ou traumatismo, o distúrbio primário é a diminuição do volume sangüíneo circulante e, conseqüentemente, redução acentuada do retorno venoso e da pressão venosa central com posterior diminuição do débito cardíaco e da pressão arterial.

O cérebro é o órgão que menos sofre interferência devido a variações sistêmicas da volemia, pois seu fluxo sangüíneo tem regulação local. Durante um estado de hipotensão, pode ocorrer vasoconstrição da microcirculação cerebral e diminuir a perfusão. No entanto, tem sido demonstrado que existem variações significativas no fluxo sangüíneo em diferentes regiões do cérebro, em resposta à hipovolemia, resultando em redistribuição do mesmo (Raiser, 1995).

O isofluorano (éter 1-cloro-2, 2, 2-trifluoroetildifluorometilico) é um anestésico volátil amplamente utilizado, com concentração alveolar mínima (CAM) de 1,39, em cães (Kazama e Ikeda, 1988), que lhe confere potência anestésica relativamente alta. Seu baixo coeficiente de solubilidade sangue-gás $(1,46)$ permite indução e recuperação anestésicas rápidas, proporcionando maior controle da profundidade da anestesia (Hall et al., 2001). Além disso, possui baixa taxa de biotransformação, de aproximadamente $0,2 \%$, reduzindo a capacidade de produzir nefrotoxicidade (Oliva, 2002).

Assim como os demais anestésicos inalatórios, o isofluorano causa alterações dependentes da dose sobre o sistema cardiovascular. Em concentrações clínicas, tende a preservar o débito cardíaco, uma vez que a diminuição no volume sistólico é compensada pelo aumento na freqüência cardíaca (Omoigui, 1998). Além disso, esse agente não sensibiliza o miocárdio à ação das catecolaminas (Tucker et al., 1974). As diminuições na pressão arterial ocorrem principalmente devido à redução significativa na resistência vascular periférica (Omoigui, 1998).

A redução do metabolismo cerebral está intimamente relacionada com a diminuição da atividade elétrica cerebral, ocorrendo apenas em presença de concentrações mais elevadas do fármaco (Detsch et al., 1998). Seus efeitos sobre a pressão intracraniana e perfusão cerebral observados em diversos estudos realizados em situações de injúria cerebral demonstraram que o fármaco apresenta efeitos benéficos quando comparado a outros anestésicos (Goren et al., 2001).

Com este estudo objetivou-se avaliar as possíveis alterações hemodinâmicas e intracranianas durante a hemorragia aguda, em cães anestesiados com isofluorano, além de verificar a influência do anestésico volátil no mecanismo de auto-regulação cerebral.

\section{MATERIAL E MÉTODOS}

Este trabalho foi aprovado pelo Comitê de Ética e Bem Estar Animal da Faculdade de Ciências Agrárias e Veterinárias - UNESP, Campus de Jaboticabal.

Foram utilizados 20 cães adultos, machos e fêmeas, sem raça definida, excluindo-se fêmeas prenhes ou em estro, e considerados hígidos após exames clínicos e laboratoriais. A média de peso dos animais foi de $11,12 \pm 3,89 \mathrm{~kg}$.

Após jejum alimentar de oito e hídrico de duas horas, induziu-se a anestesia geral nos animais com isofluorano ${ }^{1}$ por meio de máscara naso-oral vedada, a $3,5 \mathrm{~V} \%$, mensurada em analisador de gases $^{2}$, diluído em fluxo total de $150 \mathrm{ml} / \mathrm{kg} / \mathrm{min}$ de oxigênio $\left(\mathrm{O}_{2}\right)$ a $100 \%$, com o uso de circuito anestésico com reinalação parcial de gases ${ }^{3}$, dotado de vaporizador

${ }^{1}$ Forane, Abbott Laboratórios do Brasil Ltda. - Rio de Janeiro, Brasil.

${ }^{2}$ Dixtal, mod. DX 2010 - Manaus, Brasil.

${ }^{3}$ Ohmeda, mod. Excel 210SE, Datex Ohmeda - Miami, EUA. 
calibrado $^{4}$. Em seguida, realizou-se a intubação orotraqueal com sonda de Magill, que foi acoplada ao aparelho de anestesia inalatória, reajustando-se o vaporizador para $2,1 \mathrm{~V} \%$ e fluxo diluente total de $30 \mathrm{ml} / \mathrm{kg} / \mathrm{min}$ de $\mathrm{O}_{2}$ a $100 \%$.

Todos os animais foram mantidos em decúbito lateral direito sobre colchão térmico ativo ${ }^{5}$ na tentativa de manter a temperatura corporal estável.

Realizou-se uma incisão na pele da face interna do membro pélvico direito, para exposição da artéria femoral e introdução de cateter para mensuração da pressão arterial. A veia femoral também foi exposta para a colocação do cateter de Swan-Ganz ${ }^{6}$, para mensuração do débito cardíaco. Além disso, foi implantado cirurgicamente um cateter de fibra óptica ${ }^{7}$ na superfície do córtex cerebral esquerdo, usando-se kit de acesso ${ }^{8}$. Imediatamente após, o cateter foi conectado ao monitor digital de pressão intracraniana ${ }^{9}$ para aferição desta variável.

Após instrumentação, induziu-se a hemorragia aguda nos animais retirando-se o sangue da circulação por meio do cateter posicionado na artéria femoral. Foi coletado volume total de $35 \mathrm{ml} / \mathrm{kg}$ de sangue na velocidade de $20 \mathrm{ml} / \mathrm{min}$ com o uso de seringas plásticas descartáveis de $60 \mathrm{ml}$ após o preenchimento do espaço morto da seringa com heparina $(1.000 \mathrm{U} / \mathrm{ml})$ para evitar a coagulação.

As mensurações foram realizadas imediatamente antes da hipovolemia (M0), 3 minutos após a retirada do volume total de sangue calculado (M1), 10 minutos após M1 (M2), 20 minutos após M2 (M3) e 30 minutos após M3 (M4). Os parâmetros mensurados nos tempos de avaliação foram: pressão intracraniana (PIC) e temperatura intracraniana (TIC), por mensuração direta em monitor de pressão intracraniana. A pressão de perfusão cerebral (PPC) foi obtida por relação matemática, subtraindo-se do valor da PAM o valor da PIC. As pressões arteriais sistólica (PAS), diastólica (PAD) e média (PAM) foram obtidas

${ }^{4}$ Ohmeda, mod. Isotec 5, Datex Ohmeda - Miami, EUA.

${ }^{5}$ Gaymar, mod. TP 500 - Londres, Inglaterra.

${ }^{6}$ 132-5F Pediátrico, Edwards Lifesciences LLC - Califórnia, EUA.

${ }^{7}$ Intracranial pressure, temperature monitoring, mod.1104BT, Integra Neurocare Camino Labs - San Diego, EUA.

${ }^{8}$ Cranial access kit, mod. 5H-ITH-2, Integra Neurocare Camino Labs, San Diego - EUA.

${ }^{9}$ Integra, mod. MPM-1, Integra Neurocare Camino Labs San Diego, EUA. utilizando monitor multiparamétrico ${ }^{10}$ cujo sensor foi adaptado em cateter, introduzido na artéria femoral. A freqüência cardíaca (FC) foi obtida por eletrocardiógrafo computadorizado ${ }^{11}$ ajustado para leitura na derivação DII.

O débito cardíaco (DC) foi mensurado com dispositivo microprocessado ${ }^{12}$, pela técnica de termodiluição, administrando $3 \mathrm{ml}$ de solução de $\mathrm{NaCl}$ a $0,9 \%$ resfriada $\left(0-3^{\circ} \mathrm{C}\right)$ no ramo destinado à leitura da PVC do cateter de Swan-Ganz. A mensuração foi realizada em triplicata, empregando-se média aritmética para a determinação da variável.

O índice cardíaco (IC) foi obtido por relação matemática, dividindo-se o valor do DC pela área de superfície corpórea (ASC) $\mathrm{em}^{2}$. O índice sistólico (IS) foi obtido empregando relação matemática, dividindo-se o valor do VS pela ASC. A pressão venosa central (PVC) e a pressão da artéria pulmonar (PAP) foram obtidas em monitor multiparamétrico ${ }^{12}$, cujo sensor foi adaptado ao cateter de Swan-Ganz.

A freqüência respiratória (FR) e a pressão parcial de dióxido de carbono ao final da expiração $\left(\mathrm{ETCO}_{2}\right)$ foram obtidas pelo monitor multiparamétrico ${ }^{13}$, cujo sensor foi adaptado à sonda orotraqueal. A saturação de oxihemoglobina $\left(\mathrm{SpO}_{2}\right)$ foi obtida por leitura direta em monitor multiparamétrico $^{13}$, sendo o sensor conectado na língua do animal. A temperatura corpórea (T) foi obtida por monitor multiparamétrico ${ }^{13}$, cujo sensor foi introduzido no esôfago e posicionado próximo à base cardíaca. Ao término do período experimental, realizou-se a reinfusão do sangue coletado de cada animal.

A avaliação estatística das variáveis foi efetuada pela análise de variância seguida pelo Teste Tukey, com nível de significância de 5\% $(\mathrm{P}<0,05)$

\section{RESULTADOS}

Os valores (média \pm desvio-padrão) de todos os parâmetros estudados estão dispostos na Tab. 1 .

${ }^{10}$ Dixtal, mod. DX 2010, Módulo de PA invasiva, Manaus, AM, Brasil.

${ }^{11}$ TEB, mod. ECGPC software versão 1.10, São Paulo, SP, Brasil.

${ }^{12}$ Dixtal, mod. DX 2010, Módulo de débito cardíaco, Manaus, AM, Brasil.

${ }^{13}$ Dixtal, mod. DX 2010, Manaus, AM, Brasil. 
Tabela 1. Valores médios e desvios-padrão das variáveis intracranianas e hemodinâmicas obtidas em cães submetidos ao choque hipovolêmico e anestesiados com isofluorano

\begin{tabular}{cccccc}
\hline \multirow{2}{*}{ Variável } & \multicolumn{5}{c}{ Momento } \\
\cline { 2 - 6 } & M0 & M1 & M2 & M3 & M4 \\
\hline PIC & $15 \pm 5,4 \mathrm{a}$ & $11 \pm 4,6 \mathrm{~b}$ & $12 \pm 4,3 \mathrm{ab}$ & $14 \pm 4,2 \mathrm{ab}$ & $15 \pm 3,9 \mathrm{a}$ \\
PPC & $53 \pm 13,1 \mathrm{a}$ & $28 \pm 16,0 \mathrm{~b}$ & $29 \pm 14,7 \mathrm{~b}$ & $37 \pm 15,8 \mathrm{~b}$ & $39 \pm 10,7 \mathrm{~b}$ \\
TIC & $37,9 \pm 0,8$ & $37,8 \pm 0,9$ & $37,8 \pm 0,9$ & $37,8 \pm 0,9$ & $37,8 \pm 1,0$ \\
FC & $115 \pm 16,4$ & $107 \pm 23,8$ & $114 \pm 32,0$ & $120 \pm 25,7$ & $123 \pm 19,8$ \\
PAS & $89 \pm 16,1 \mathrm{a}$ & $57 \pm 28,4 \mathrm{c}$ & $60 \pm 20,5 \mathrm{bc}$ & $71 \pm 21,0 \mathrm{abc}$ & $78 \pm 18,5 \mathrm{ab}$ \\
PAD & $55 \pm 13,2 \mathrm{a}$ & $30 \pm 9,15 \mathrm{c}$ & $33 \pm 13,7 \mathrm{bc}$ & $40 \pm 12,4 \mathrm{bc}$ & $43 \pm 14,5 \mathrm{~b}$ \\
PAM & $68 \pm 12,1 \mathrm{a}$ & $39 \pm 17,1 \mathrm{c}$ & $41 \pm 15,2 \mathrm{c}$ & $51 \pm 14,4 \mathrm{bc}$ & $54 \pm 9,9 \mathrm{~b}$ \\
IC & $4,2 \pm 1,0 \mathrm{a}$ & $1,6 \pm 0,7 \mathrm{~d}$ & $1,9 \pm 0,7 \mathrm{~cd}$ & $2,4 \pm 0,7 \mathrm{bc}$ & $2,7 \pm 0,6 \mathrm{~b}$ \\
IS & $37,0 \pm 9,0 \mathrm{a}$ & $15,5 \pm 5,8 \mathrm{c}$ & $17,8 \pm 6,7 \mathrm{bc}$ & $20,5 \pm 6,2 \mathrm{bc}$ & $22,1 \pm 6,1 \mathrm{~b}$ \\
PVC & $3 \pm 3,5 \mathrm{a}$ & $0 \pm 3,11 \mathrm{~b}$ & $0 \pm 3,6 \mathrm{~b}$ & $0 \pm 3,1 \mathrm{~b}$ & $0 \pm 3,5 \mathrm{~b}$ \\
PAP & $12 \pm 4,1 \mathrm{a}$ & $5 \pm 3,3 \mathrm{~b}$ & $6 \pm 3,2 \mathrm{~b}$ & $6 \pm 2,8 \mathrm{~b}$ & $7 \pm 3,3 \mathrm{~b}$ \\
TC & $38,1 \pm 0,6$ & $38,0 \pm 0,5$ & $38,0 \pm 0,6$ & $38,0 \pm 0,6$ & $37,8 \pm 0,8$ \\
$\mathrm{ETCO}_{2}$ & $46 \pm 8,7$ & $39 \pm 6,1$ & $41 \pm 7,2$ & $42 \pm 7,6$ & $45 \pm 8,7$ \\
$\mathrm{FR}$ & $10 \pm 6,1$ & $14 \pm 6,4$ & $14 \pm 6,8$ & $14 \pm 7,0$ & $13 \pm 7,1$ \\
$\mathrm{SpO}_{2}$ & $98 \pm 1,3$ & $98 \pm 1,5$ & $98 \pm 1,4$ & $98 \pm 1,1$ & $98 \pm 1,1$ \\
\hline
\end{tabular}

Médias seguidas de letras distintas na linha diferem entre si pelo teste Tukey $(\mathrm{P}<0,05)$. PIC: pressão intracraniana $(\mathrm{mmHg})$; PPC: pressão de perfusão cerebral $(\mathrm{mmHg})$; TIC: temperatura intracraniana $\left({ }^{\circ} \mathrm{C}\right)$; FC: freqüência cardíaca (bat/min); PAS: pressão arterial sistólica (mmHg); PAD: pressão arterial diastólica (mmHg); PAM: pressão arterial média $(\mathrm{mmHg})$; IC: índice cardíaco $\left(\mathrm{L} / \mathrm{min}^{*} \mathrm{~m}^{2}\right)$; IS: índice sistólico (mL/bat*m²); PVC: pressão venosa central (mmHg); PAP: pressão da artéria pulmonar $(\mathrm{mmHg})$; $\mathrm{ETCO}_{2}$ : pressão parcial de dióxido de carbono ao final da expiração $(\mathrm{mmHg})$; FR: freqüência respiratória (mov/min); $\mathrm{SpO}_{2}$ : saturação de oxihemoglobina (\%); T: temperatura corpórea $\left({ }^{\circ} \mathrm{C}\right)$.

Imediatamente após a indução de hipovolemia, notou-se redução significativa da PIC, sendo possível observar aumento gradativo nos momentos subseqüentes, até o retorno ao valor basal na última aferição (Fig. 1). Verificou-se redução significativa da PPC em M1 quando comparada ao momento basal, permanecendo nesse patamar até o momento subseqüente. Embora não significativo, a partir desse momento, observou-se discreto incremento dessa variável, sem, entretanto, atingir o valor inicial.

Houve diminuição significativa das pressões arteriais no momento imediatamente após a hemorragia, quando comparados ao valor basal, com subseqüente aumento gradativo. As médias da PAS em M1 e M2 foram significativamente menores que M0, sendo que, a partir de M2, os valores tenderam a retornar ao valor basal. Tanto a PAD como a PAM indicaram que, em todos os momentos, os valores registrados foram significativamente menores que o valor basal. Entretanto, durante o transcorrer do período experimental, a PAD e a PAM foram se elevando, sendo que as médias no M4 foram significativamente maiores que no M1 (Fig. 2).
A PVC diminuiu a partir de M1 quando comparado a M0, porém essa redução foi somente significativa em M3, momento em que esta variável apresentou valor mais baixo, comparando com os demais momentos (Fig. 3). A PAP diminuiu de maneira significativa após a indução da hipovolemia, quando comparado ao valor inicial, sendo que, nos momentos seguintes, os valores permaneceram inferiores a M0 até o término do período experimental.

Em relação ao IC, observou-se que todo o período subseqüente ao momento inicial apresentou valores significativamente inferiores a este. Entretanto, a partir de M1, o IC foi aumentando, sendo que M3 e M4 foram maiores que M1 (Fig. 4).

Quanto ao IS, verificou-se que todos os momentos foram significativamente inferiores em relação ao valor basal. Porém, do mesmo modo que o IC, o IS foi retornando aos valores iniciais ao longo do período experimental, sendo que M4 apresentou valores acima de M1, não alcançando, porém, os valores iniciais (Fig. 5). 


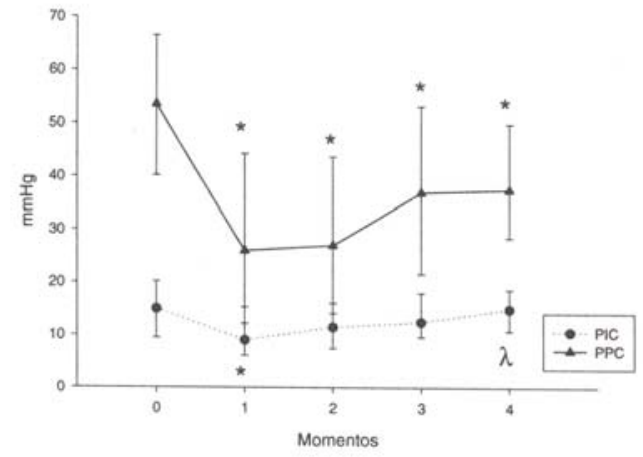

Figura 1. Média \pm desvio-padrão da pressão intracraniana (PIC) e pressão de perfusão cerebral (PPC) em cães com hemorragia aguda, anestesiados com isofluorano. Diferença significativa $(\mathrm{P}<0,05)$ quando comparado com M0 $(*)$; quando comparado com M1 $(\lambda)$.

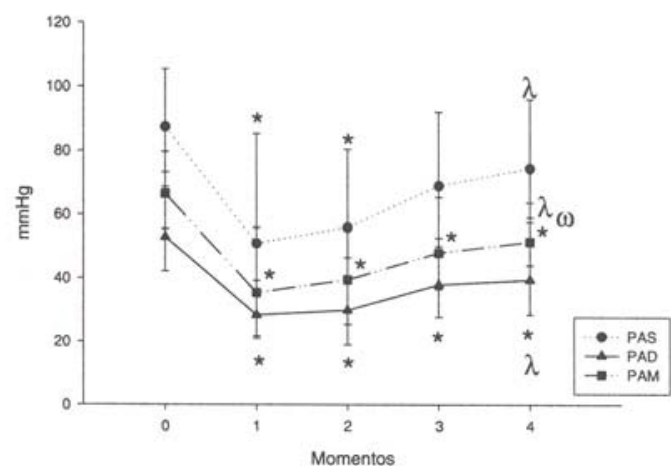

Figura 2. Média \pm desvio-padrão das pressões arteriais sistólica (PAS), diastólica (PAD) e média (PAM) em cães com hemorragia aguda, anestesiados com isoflurano. Diferença significativa $(\mathrm{P}<0,05)$ quando comparado com M0 $(*)$; quando comparado com M1 $(\lambda)$; quando comparado com M2 $(\omega)$.

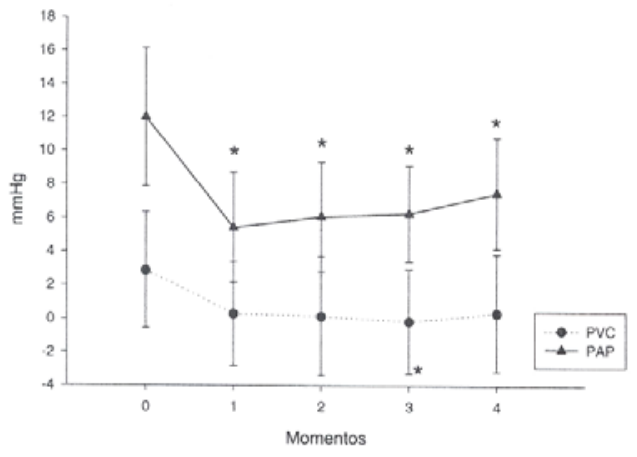

Figura 3. Média desvio-padrão da pressão venosa central (PVC) e pressão da artéria pulmonar (PAP) em mmHg. Diferença significativa $(\mathrm{P}<0,05)$ quando comparado com $\mathrm{M} 0$ (*).

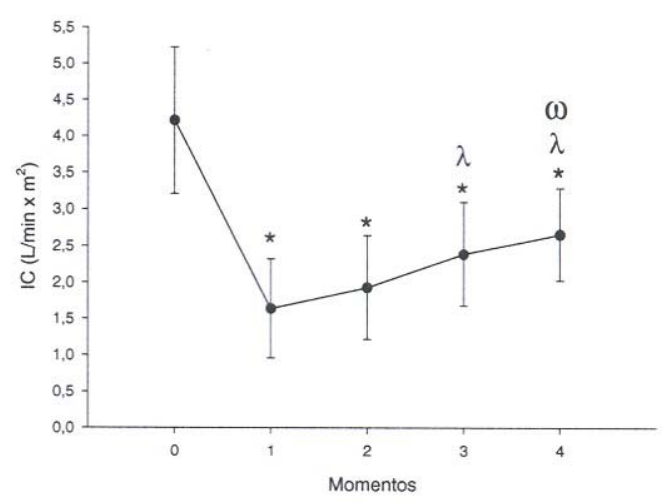

Figura 4. Média \pm desvio-padrão do índice cardíaco (IC) em $1 / \mathrm{min}^{*} \mathrm{~m}^{2}$. Diferença significativa $(\mathrm{P}<0,05)$ quando comparado com M0 $(*)$; quando comparado com M1 $(\lambda)$; quando comparado com M2 $(\omega)$.

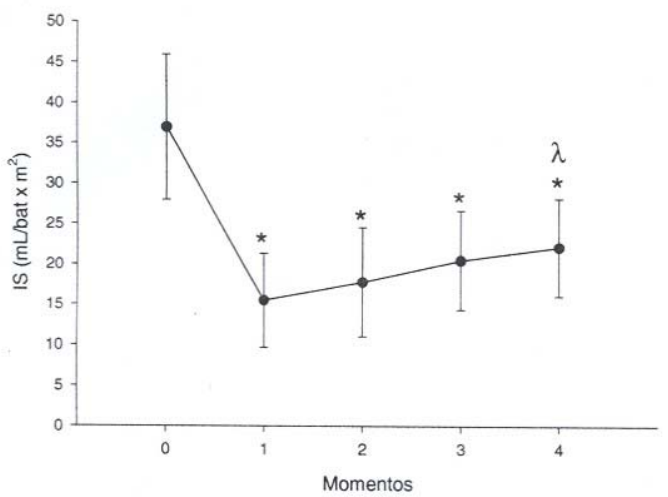

Figura 5. Média \pm desvio-padrão do índice sistólico (IS) em $\mathrm{ml} / \mathrm{bat} / \mathrm{m}^{2}$. Diferença significativa $(\mathrm{P}<0,05)$ quando comparado a M0 $(*)$; quando comparado a M1 $(\lambda)$.

As demais variáveis (TIC, TC, FC, $\mathrm{ETCO}_{2}, \mathrm{FR}$ e $\mathrm{SpO}_{2}$ ) não apresentaram diferenças significativas entre os momentos do período experimental.

\section{DISCUSSÃO}

Os valores relativos às pressões arteriais sistólica, diastólica e média e índices cardíaco e sistólico, medidos três minutos após o término da hemorragia, apresentaram-se reduzidos, devido, provavelmente, à alteração do volume sangüíneo e à conseqüente redução do retorno venoso. Achados semelhantes foram descritos por Meletti e Módolo (2003), após hemorragia progressiva de $50 \%$ do volume total do sangue de cães sem raça definida. Além disso, observou-se que, nos 
momentos seguintes, as médias dessas variáveis tenderam a aumentar, comportando-se de maneira semelhante à FC que, embora não significativo, apresentou redução de suas médias três minutos após a hemorragia, com tendência ao aumento nos momentos subseqüentes. Höhne et al. (2004) analisaram os efeitos da hemorragia em cães anestesiados com isofluorano e óxido nitroso e observaram que houve aumento da FC após a hemorragia, sendo esse efeito decorrente do efeito vagolítico do isofluorano (Picker et al., 2001).

As alterações observadas neste estudo provavelmente ocorreram devido ao mecanismo compensatório, em resposta à perda sangüínea. Segundo Martel (2002) e Kirby (2004), uma vez que a hipovolemia foi instalada, ocorre vasoconstrição periférica por estímulo simpático, principalmente da vasculatura esplênica e músculo-esquelética, além de taquicardia e aumento da contratilidade do miocárdio, com favorecimento da redistribuição em benefício da circulação cerebral, coronariana e hepática (Díaz, 2004).

Höhne et al. (2004), ao estudarem as possíveis alterações que a hemorragia poderia causar durante anestesia com isofluorano associado ao óxido nitroso, em cães, observaram que houve redução da PAM e DC cinco minutos após a hemorragia.

O isofluorano apresenta efeito depressor cardiovascular (Park, 2002). Avram et al. (2000) estudaram os efeitos farmacocinéticos desse anestésico em cães e relataram redução da pressão arterial e do débito cardíaco. Janssen et al. (2004) verificaram o mesmo efeito depressor em ratos. Assim, a redução das médias desses parâmetros, neste estudo, pode ter sido conseqüência da interação dos efeitos promovidos pela hemorragia e pelo isofluorano sobre a função cardiovascular.

A redistribuição sangüínea por vasoconstrição periférica contribuiu com o incremento da PPC 10 minutos após a hemorragia. Jones e Wardrop (2000) afirmaram que um dos mecanismos compensatórios durante a hemorragia seria o fornecimento do aporte sangüíneo apropriado, principalmente ao cérebro e coração. Assim, o aumento do fluxo sangüíneo cerebral observado neste estudo foi responsável pela elevação dos valores de PIC, a partir de 10 minutos após o término da hemorragia.

Sabe-se que a PPC reflete a perfusão sangüínea do tecido cerebral e é obtida por relação matemática, subtraindo-se do valor da PAM o valor da PIC, sendo, portanto, influenciada diretamente por variações desses parâmetros. Em cães, quando os valores de PPC ficam abaixo de $40 \mathrm{mmHg}$, há risco de ocorrência de isquemia cerebral (Sulek, 1997).

Neste estudo, os animais provavelmente apresentaram redução da perfusão sangüínea cerebral durante a hemorragia aguda, uma vez que os valores de PPC apresentaram-se abaixo do fisiológico para a espécie (Sulek, 1997). O fato de a PPC estar reduzida demonstrou que o fluxo sangüíneo cerebral não permaneceu em níveis adequados, prejudicando a perfusão cerebral. Isso se deve provavelmente à acentuada redução da PAM, pois, segundo Sponheim et al. (2003), a influência desse parâmetro na PPC é três a quatro vezes mais significativa do que a da PIC. Porém, não se pode descartar a hipótese de que o isofluorano possa ter auxiliado na hipotensão, pois, segundo Fraga et al. (2003), em um estudo com pacientes com tumores cerebrais, há redução da PPC com a administração de isofluorano devido à diminuição da PAM ocasionada pelo fármaco.

Sasaki et al. (2005) observaram que o isofluorano não preveniu a despolarização anóxica do hipocampo em ratos, como é esperado quando se utiliza o tiopental, o qual, como barbiturato, promove efeitos profiláticos nos casos de isquemia. Estudos recentes sugerem que uma combinação de bloqueadores dos canais de sódio e cálcio, antagonistas dos receptores NMDA e não-NMDA, pode efetivamente prevenir a despolarização anóxica (ou isquemia cerebral) (Somjen, 2001). Porém, o isofluorano é incapaz de atenuar as alterações no influxo de potássio e sódio durante a anóxia (Kass et al., 1997). Assim, neste estudo, a isquemia, ocasionada pela redução da PPC devido à hipovolemia, pode ter sido incrementada pela concomitante administração do anestésico inalatório.

Segundo Bagley et al. (1995), o mecanismo de auto-regulação cerebral é ativado quando um dos componentes do conteúdo intracraniano, que 
inclui o parênquima cerebral, líquido cerebroespinhal, sangue e meninges, sofre alguma alteração, havendo, portanto, a necessidade de outros se modificarem para que a PIC permaneça constante. Esse mecanismo provavelmente foi ativado em decorrência da hipovolemia induzida, uma vez que houve incremento dessa variável a partir de 10 minutos após a hemorragia, fazendo com que os valores retornassem aos iniciais de controle.

Artru (1994) relatou que o isofluorano eleva a PIC, devido à vasodilatação cerebral, e, conseqüentemente, diminui a resistência vascular cerebral e aumenta o fluxo sangüíneo cerebral de maneira dependente da dose (Werner e Hänel, 1998). Entretanto, Krayer (2000) relatou que, quando se utilizam concentrações baixas do anestésico, o mecanismo de auto-regulação permanece intacto, e os efeitos vasodilatadores do fármaco são mínimos. Esse fato pode ter contribuído para a manutenção dos valores da PIC, que permaneceram estáveis durante aproximadamente 30 minutos, tempo necessário para a instrumentação dos animais, havendo redução das médias somente após a hipovolemia, que foi responsável pela ativação do mecanismo de auto-regulação cerebral.

Do mesmo modo, Fraga et al. (2003) observaram que tanto o isofluorano quanto o desfluorano, quando utilizados em concentrações clínicas, não promoveram alterações na PIC em pacientes com preservação da elasticidade cerebral. Holmström e Akeson (2004) verificaram que o desfluorano promove maior vasodilatação cerebral e valores maiores de PIC quando comparado ao isofluorano e sevofluorano, porém o aumento do fluxo sangüíneo cerebral, resultante do efeito vasodilatador dos anestésicos, não foi capaz de promover alterações significativas na PIC.

Durante o choque hemorrágico, há redução do fluxo sangüíneo dos músculos esqueléticos, incluindo os músculos respiratórios (Díaz, 2004). Além disso, há necessidade de aumentar o volume-minuto na tentativa de manter a ventilação alveolar adequada (Martel, 2002). Assim, é freqüente observar relatos de taquipnéia, hipoxia e redução dos níveis de oxigênio arterial (Martel, 2002; Díaz, 2004). Neste estudo, não foi possível observar diferenças importantes na FR, $\mathrm{ETCO}_{2}$ e $\mathrm{SpO}_{2}$.
Kurita et al. (2005) estudaram a influência da hipovolemia em suínos anestesiados com isofluorano e observaram que, após a retirada de $20 \%$ do volume total de sangue, houve redução significativa da pressão parcial de dióxido de carbono. Esse resultado não foi observado neste estudo.

O dióxido de carbono é um potente vasodilatador cerebral, e a hipercapnia prejudica a auto-regulação cerebral (Haggendal e Johansson, 1965). Por esse motivo, a hiperventilação é normalmente empregada durante anestesia em procedimentos neurocirúrgicos (McCulloch et al., 2005). Esses autores observaram que a hipocapnia pode restabelecer a auto-regulação cerebral, antagonizando o efeito vasodilatador do isofluorano. Neste estudo, não se observou hipercapnia, portanto, provavelmente não houve interferência do dióxido de carbono na autoregulação cerebral. Da mesma forma, não foi possível observar o seu restabelecimento com o auxílio da hiperventilação, uma vez que esse fenômeno não ocorreu durante $\mathrm{o}$ período experimental.

A temperatura corpórea foi mantida em níveis fisiológicos (Robinson, 1992) com o uso de colchão térmico ativo, o que justifica também a manutenção da TIC, já que esta acompanhou a variação da primeira. Entretanto, Heinius et al. (2002), ao estudarem suínos em choque hemorrágico submetidos à hipotermia induzida, observaram que a redução da temperatura corpórea não agravou a hemodinâmica e que a diminuição da taxa metabólica auxiliou na estabilização da taxa de extração de oxigênio, sugerindo que a hipotermia pode apresentar efeito protetor em pacientes com choque hemorrágico.

Segundo Cortopassi (2002), um dos melhores parâmetros para avaliação da volemia é a PVC, pois ela é afetada pela pré-carga cardíaca, a qual é amplamente dependente do volume sangüíneo. Observou-se que a PVC apresentou redução de seus valores após a exsangüinação, provavelmente em função do decréscimo do volume circulante (Muir, 1998) e, conseqüentemente, redução da perfusão periférica (Raiser, 1995). O mesmo resultado foi observado por Potas e Dampney (2004), ao induzirem hipovolemia em ratos, pois à medida que o sangue era retirado, a pressão venosa central diminuía gradativamente. Do mesmo modo, Kurita et al. (2005) observaram redução 
significativa da PVC após a retirada de $20 \%$ do volume total de sangue de suínos anestesiados com isofluorano. Neste estudo, foi possível verificar que, devido aos mecanismos compensatórios, somente 60 minutos após a hemorragia observou-se que as médias da PVC tenderam a retornar aos valores basais.

A pressão média da artéria pulmonar é rotineiramente empregada na clínica de emergência para estimar a pressão de enchimento do ventrículo esquerdo (Shoemaker, 1989; Mark, 1998), revestindo-se de importância na avaliação da pré-carga e da função ventricular esquerda (Santos, 2003). Assim, a redução das médias dessa variável foi conseqüente à diminuição da pressão arterial ocasionada pela depleção do volume sangüíneo, e o efeito depressor cardiovascular do isofluorano (Park, 2002) pode ter contribuído neste fenômeno.

\section{CONCLUSÕES}

Conclui-se que a hemorragia aguda promoveu redução acentuada nos parâmetros hemodinâmicos, sendo possível verificar a ativação dos mecanismos compensatórios do choque hemorrágico, uma vez que todos os animais recuperaram-se posteriormente. Além disso, houve redução da perfusão sangüínea cerebral e ativação do mecanismo de autoregulação cerebral, conseqüentes à hipovolemia associada ao isofluorano.

\section{REFERÊNCIAS BIBLIOGRÁFICAS}

ARTRU, A.A. Intracranial volume/pressure relashionship during desflurane anesthesia in dogs: Comparison with isoflurane and thiopental/halotane. Anesth. Analg., v.79, p.751-760, 1994.

AVRAM, M.J.; KREJCIE, T.C.; NIEMANN, C.U. et al. Isoflurane alters the recirculatory pharmacokinetics of physiologic markers. Anesthesiology, v.92, p.17571768, 2000.

BAGLEY, R.S.; KEEGAN, R.D.; GREENE, S.A. et al. Pathologic effects in brain after intracranial pressure monitoring in clinically normal dogs, using a fiberoptic monitoring system. Am. J. Vet. Res., v.56, p.1475-1478, 1995.

CLARCK, D.R. Tratamento do choque circulatório. In: BOOTH, N.H.; McDONALD, L.E. Farmacologia e terapêutica em veterinária. 6.ed. Rio de Janeiro: Guanabara-Koogan, 1992. p.449-454.

CORTOPASSI， S.R.G. Anestesia pediátrica. In: FANTONI, D.T.; CORTOPASSI, S.R.G. Anestesia em cães e gatos. 1.ed. São Paulo: Roca, 2002. p.216221.

DETSCH, O.; SCHNEIDER, G.; HAPFELMEIER, G. et al. Increasing isoflurane may induce paradoxical increases in the EEG bispectral index (BIS). Anesthesiology, v.89, suppl.3A, p.363A, 1998.

DÍAZ, J.M. Anestesia en el paciente en shock hemorrágico. Servicio de Anestesiología, Hospital de Urgência, Asistencia Pública. Disponível em: $<$ http://usuarios.vtr.net/ anestesi/clases/hemorragia/sh ock_hemorragico.pdf $>$. Acesso em: 15 dez. 2004.

FRAGA, M.; RAMA-MACEIRAS, P.; RODIÑO, S. et al. The effects of isoflurane and desflurane on intracranial pressure, cerebral perfusion pressure, and cerebral arteriovenous oxygen content difference in normocapnic patients with supratentorial brain tumors. Anesthesiology, v.98, p.1085-1090, 2003.

GOREN, S.; KAHVECI, N.; ALKAN, T. et al. The effects of sevoflurane and isoflurane on intracranial pressure and cerebral perfusion pressure after diffuse brain injure in rats. J. Neurosurg. Anesthesiol., v.13, p.113-119, 2001.

HAGGENDAL, E.; JOHANSSON, B. Effects of arterial carbon dioxide tension and oxygen saturation on cerebral blood flow autoregulation in dogs. Acta Physiol. Scan., v.258, suppl., p.27-53, 1965.

HALL, L. W.; ClARKE, K. W.; TRIM, C. M. General pharmacology of the inhalation anaesthetics. In: . Veterinary anaesthesia. 10.ed. London: W.B. Saunders, 2001. p.133-147.

HEINIUS, G.; WLADIS, A.; HAHN, R.G. et al. Induced hypothermia and rewarming after hemorrhagic shock. J. Surg. Res., v.108, p.7-13, 2002.

HÖHNE, C.; VOGLER, P.; FRERKING, I. et al. Hemorrhage during isoflurane-nitrous oxide anesthesia. Anesthesiology, v.100, p.885-893, 2004.

HOLMSTRÖM, A.; AKESON, J. Desflurane increases intracranial pressure more and sevoflurane less than isoflurane in pigs subjected to intracranial hypertension. J. Neurosurg. Anesthesiol., v.16, p.136143, 2004.

JANSSEN, B.J.; De-CELLE, T.; DEBETS, J.J. et al. Effects of anesthetics on systemic hemodynamics in mice. Am. J. Physiol. Heart Cir. Physiol., v.287, p.H1618-1624, 2004.

JONES, J.G.; WARDROP, C.A. Measurement of blood volume in surgical and intensive care practice. Br. J. Anaesth., v.84, p.226-235, 2000. 
KASS, I.S.; AMORIM, P.; CHAMBERS, G. et al. The effect of isoflurane on biochemical changes during and electrophysiological recovery after anoxia in rat hippocampal slices. J. Neurosurg. Anesthesiol., v. 9, p.280-286, 1997.

KAZAMA, T.; IKEDA, K. Comparison of MAC and the rate of rise of alveolar concentration of sevoflurane with halothane and isoflurane in the dog. Anesthesiology, v.68, p.435-438, 1988.

KIRBY, R. Shock and resuscitation parts I and II. In: WORLD SMALL ANIMAL AND VETERINARY CONGRESS, 29., 2004, Rhodes, Greece. Proceedings... Rhodes-Greece, 2004. 1 CD-ROM.

KRAYER, S. Anesthesia for interventional neuroradiology. Curr. Opin. Anaesthesiol., v.13, p.421-427, 2000

KURITA, T.; MORITA, K.; FUKUDA, K. et al. Influence of hypovolemia on the electroencephalographic effect of isoflurane in a swine model. Anesthesiology, v.102, p.948-953, 2005.

MARK, J.B. Atlas of cardiovascular monitoring. New York: Churchill Livingstone, 1998. 362p.

MARTEL, M.J. Hemorrhagic shock. J. Obstet. Gynaecol. Can., v.24, p.504-511, 2002.

McCULLOCH, T.J.; BOESEL, T.W.; LAM, A.M. The effect of hypocapnia on the autoregulation of cerebral blood flow during administration of isoflurane. Anesth. Analg., v.100, p.1463-1467, 2005.

MELETTI, J.F.A.; MÓDOLO, N.S.P. Comportamento hemodinâmico e metabólico do choque hemorrágico: estudo experimental no cão. Rev. Bras. Anestesiol., v.53, p.623-632, 2003

MUIR, W.W. Shock. Compendium, v.20, p.549-566, 1998.

OLIVA, V.N.L.S. Anestesia inalatória. In: FANTONI, D.T.; CORTOPASSI, S.R.G. Anestesia em cães e gatos. 1.ed. São Paulo: Roca, 2002. p.174-183.

OMOIGUI, S. Manual de drogas usadas em anestesia. 2.ed. São Paulo: Livraria Santos, 1998. 566p.

PARK, K.W. Cardiovascular effects of inhalational anesthetics. Int. Anesthesiol. Clin., v.40, p.1-14, 2002.

PICKER O.; SCHEEREN, T.W.L.; ARNDT, J.O. Inhalation anesthetics increase heart rate by decreasing cardiac vagal activity in dogs. Br. J. Anaesth., v.87, p.748-754, 2001

POTAS, J.R.; DAMPNEY, R.A.L. Evidence that venoconstriction reverses the phase II sympathoinhibitory and bradycardic response to haemorrhage. Auton. Neurosc.: Basic Clin., v.111, p.1-6, 2004.

RAISER, A.G. Choque. In: Patologia cirúrgica veterinária. Santa Maria, 1995. v.1, cap.3, p.47-100.

ROBINSON, E. Termorregulação. In: CUNNINGHAM, J.G. Tratado de fisiologia veterinária. 1.ed. Rio de Janeiro: Guanabara Koogan, 1992. p.427435.

SANTOS, P.S.P. Efeitos hemodinâmicos, eletrocardiográficos e hemogasométricos do butorfanol em cães anestesiados pelo desfluorano. 2003. 141f. Tese (Doutorado em Cirurgia Veterinária)

- Faculdade de Ciências Agrárias e Veterinárias, Universidade Estadual Paulista, Jaboticabal, SP.

SASAKI, R.; HIROTA, K.; ROTH, S.H. et al. Anoxic depolarization of rat hippocampal slices is prevented by thiopental but not by propofol or isoflurane. $\mathrm{Br} . \mathrm{J}$. Anaesth., v.94, p.486-491, 2005.

SHOEMAKER, W.C. Physiologic monitoring of the critically ill patient. In: Textbook of critical care. 2.ed. Philadelphia: W.B. Saunders, 1989. p.156171.

SOMJEN, G.G. Mechanisms of spreading depression and hypoxic spreading depression-like depolarization. Physiol. Rev., v. 81, p. 1065-1096, 2001.

SPONHEIM, S.; SKRAASTAD, O.; HELSETH, E. et al. Effects of 0.5 and 1.0 MAC isoflurane, sevoflurane and desflurane on intracranial and cerebral perfusion pressures in children. Acta Anaesthesiol. Scand., v.47, p.932-938, 2003.

SULEK, C.A. Critical care - neurosurgical critical care. In: MILLER, R.D. Atlas of anesthesia. Philadelphia: Churchill Livingstone, 1997. v.I, p.9193.

TUCKER, W.K.; RACKSTEIN, A.D.; MUNSON, E.S. Comparasion of arrhythmic dose of adrenaline, metaraminol, ephedrine and phenylephyne during isoflurane and halothane anesthesia in dogs. $B r . J$. Anaesth., v.46, p.392, 1974.

WERNER, C.; HÄNEL, F. Effects of anesthetics on cerebral blood flow and metabolism. Acta Anaesthesiol. Scand., v.42, suppl. 112, p.124-126, 1998. 